\title{
Reach Out and Touch Someone: Anticipatory Sensorimotor Processes of Active Interpersonal Touch
}

\author{
Sjoerd J. H. Ebisch ${ }^{1}$, Francesca Ferri ${ }^{2,3}$, Gian Luca Romani ${ }^{1}$, \\ and Vittorio Gallese ${ }^{2}$
}

\begin{abstract}
Anticipating the sensorimotor consequences of an action for both self and other is fundamental for action coordination when individuals socially interact. Somatosensation constitutes an elementary component of social cognition and sensorimotor prediction, but its functions in active social behavior remain unclear. We hypothesized that the somatosensory system contributes to social haptic behavior as evidenced by specific anticipatory activation patterns when touching an animate target (human hand) compared with an inanimate target (fake hand). fMRI scanning was performed during a paradigm that allowed us to isolate the anticipatory representations of active interpersonal touch while controlling for nonsocial sensorimotor processes and possible confounds because of interpersonal relationships or socioemotional valence. Active interpersonal touch was studied both as skin-to-skin contact and as object-mediated
\end{abstract}

\section{INTRODUCTION}

Interpersonal touch is a primary expression of affiliative behavior. It reflects the disposition of individuals to seek close contact between them, promoting socioemotional development, group cohesion, and reproduction (Dunbar, 2010; Gallace \& Spence, 2010; Morrison, Loken, \& Olausson, 2010). Despite the clear interactive character of interpersonal touch, psychological and neuroscientific investigations addressed it almost exclusively as a receptive experience. Gibson (1962) already emphasized the functional specificity of active touch, but research on active touch was mostly confined to the study of humanobject interactions.

Important differences exist between our tactile interactions with the animate or inanimate world. For example, whereas the former is driven by the intention to explore, manipulate, and use objects (Johansson \& Flanagan, 2009; Lederman \& Klatzky, 2009), the latter essentially has a communicative intention through somatosensory interaction with another individual (Gallace \& Spence, 2010; Hertenstein, Keltner, App, Bulleit, \& Jaskolka, 2006). From

${ }^{1}$ G. d'Annunzio University, Chieti, Italy, ${ }^{2}$ Parma University, ${ }^{3}$ University of Ottawa Institute of Mental Health Research touch. The results showed weaker deactivation in primary somatosensory cortex and medial pFC and stronger activation in cerebellum for the animate target, compared with the inanimate target, when intending to touch it with one's own hand. Differently, in anticipation of touching the human hand with an object, anterior inferior parietal lobule and lateral occipital-temporal cortex showed stronger activity. When actually touching a human hand with one's own hand, activation was stronger in medial pFC but weaker in primary somatosensory cortex. The findings provide new insight on the contribution of simulation and sensory prediction mechanisms to active social behavior. They also suggest that literally getting in touch with someone and touching someone by using an object might be approached by an agent as functionally distinct conditions.

this perspective, it can be argued that active animate and inanimate touch may not only differ in the experience of the touch itself but that active interpersonal touch may be already unique in the processes anticipating it. This study specifically aimed at clarifying the anticipatory somatosensory processes of active interpersonal touch, compared with anticipatory somatosensory processes of active object touch, by means of fMRI.

Anticipation is fundamental to action (Blakemore \& Frith, 2003; Haggard \& Clark, 2003; Wolpert \& Flanagan, 2001). During action performance, predictions are made by the brain about the sensorimotor consequences to anticipate effects and optimize performance (Prinz, 2012; Knoblich \& Flach, 2001; Wolpert, Ghahramani, \& Jordan, 1995). Several sensorimotor brain regions have been implicated in such predictions, including somatosensory and parietal cortices, cerebellum, and SMA (Haggard, 2008; Blakemore \& Sirigu, 2003). Especially relevant for social action coordination, in addition to the anticipation of one's personal sensorimotor experiences, the anticipation of others' behavior and experiences plausibly also has a clear functional value (Sebanz, Bekkering, \& Knoblich, 2006). For example, in the case of active interpersonal touch, intensity, velocity, and fine motor skills are regulated based on the expected sensation of both oneself 
and the other. It is likely to posit that sensorimotor brain circuits involved in the processing of first-person tactile experiences contribute to active interpersonal touch by anticipating not only one's own but also others' behavior and experiences for the regulation of touch performance.

Somatosensation constitutes an elementary component of both action consequences (Blakemore \& Sirigu, 2003) and social cognition (Gallese \& Ebisch, 2013; Gallese \& Sinigaglia, 2011; Keysers, Kaas, \& Gazzola, 2010). Activity in somatosensory cortices is modulated by anticipation of tactile stimuli (Carlsson, Petrovic, Skare, Petersson, \& Ingvar, 2000) and active movement (Jackson, Parkinson, Pears, \& Nam, 2011), which in turn modulates tactile stimulus processing (van Ede, de Lange, \& Maris, 2013; Jackson et al., 2011; Voss, Ingram, Wolpert, \& Haggard, 2008). Furthermore, empirical evidence consistently suggests that primary (SI, in particular BA 2) and secondary (SII) somatosensory cortices also contribute to the understanding of other individuals' tactile experiences (see, for reviews, Gallese \& Ebisch, 2013; Gallese \& Sinigaglia, 2011; Keysers et al., 2010). Some studies indicated that other regions endowed with tactile properties, like anterior inferior parietal lobule (aIPL), ventral premotor cortex, and lateral occipital-temporal cortex (IOT), could also be involved in somatosensory aspects of social perception (Morrison, Tipper, Fenton-Adams, \& Bach, 2013; Ebisch et al., 2008). Although not systematically investigated, psychological evidence supports a role of embodied simulation in the predictive coding of others' peripheral sensations (Bosbach, Cole, Prinz, \& Knoblich, 2005). Neuroimaging studies suggested that predicting the consequences of observed object-directed actions involves brain regions with somatosensory properties (Morrison et al., 2013; Ramsey, Cross, \& Hamilton, 2012).

Most somatosensory regions related to social perception also are strongly linked with motor behavior, making them plausible candidates for regulating active social touch. BA 2 and SII have direct reciprocal connections with intraparietal sulcus and aIPL, areas involved in multisensory integration as well as vicarious sensorimotor functions (Ishida, Nakajima, Inase, \& Murata, 2010; Keysers et al., 2010; Rizzolatti \& Sinigaglia, 2010; Rozzi et al., 2006; Bremmer et al., 2001; Lewis \& Van Essen, 2000; Pons \& Kaas, 1986). BA 2 also projects to primary motor cortex (Caria, Kaneko, Kimura, \& Asanuma, 1997; Kaneko, Caria, \& Asanuma, 1994a, 1994b) and has a crucial role in motor control during haptic behavior (Freund, 2003; Iwamura \& Tanaka, 1996; Hikosaka, Tanaka, Sakamoto, \& Iwamura, 1985). Furthermore, aIPL (including areas PF and PFG) and ventral premotor cortex are involved in sensorimotor coupling underlying the integration of multisensory information with motor representations for the control of goal-related motor behavior (Rozzi et al., 2006; Gallese, Fadiga, Fogassi, \& Rizzolatti, 2002; Rizzolatti, Fogassi, \& Gallese, 2002; Hyvärinen, 1982).

Hence, we hypothesized that, in the case of touching someone as well as in the case of touching something, cortical motor and somatosensory circuits likely contribute to the prediction of the sensorimotor consequences of touch performance. In particular, touch directed at another individual might be characterized by differential anticipatory neural activation patterns in brain circuits involved in somatosensation and social cognition, when compared with touch directed at inanimate targets. In addition, we speculated that actions leading to skin-toskin contact, that is, literally getting in touch with someone, and touching another individual through an object possibly might be characterized by distinct anticipatory sensory activity patterns. Skin-to-skin contact, that is, direct bodily interaction, is associated with a different intention, but also a different relevance for the personal perception of the touch, compared with an inanimate touch. By contrast, touching an animate or inanimate target mediated by an object, that is, indirect bodily interaction, only differs with respect to its intention with a more marked accentuation of the goal of the action.

To address these issues, fMRI scanning was performed in healthy participants during an experimental paradigm designed to isolate the anticipatory sensorimotor representations of social touch, while controlling for nonsocial sensorimotor processes and possible confounds because of interpersonal relationships or socioemotional valence. Interpersonal touch was studied either as skin-to-skin contact with another individual or as tactile stimulation of another individual without direct bodily contact. Whereas the former can be considered as an action resulting in a unique, shared sensory experience between two human beings, the latter concerns an action directed at inducing a sensation in the other without tactilely experiencing its sociality.

\section{METHODS}

\section{Participants}

Sixteen healthy, right-handed, young adults (age $=20$ 34 years; eight women) participated in the present experiment. All participants had normal or corrected-to normal vision capabilities. Written informed consent was obtained from all participants after full explanation of the study's procedure, in line with the Declaration of Helsinki. The experimental protocol was approved by the local institutional ethics committee. Participants were paid for their participation in the fMRI experiment.

\section{fMRI Data Acquisition}

For each participant, BOLD contrast functional imaging was performed with a Philips Achieva scanner (Andover, $\mathrm{MA})$ at $3 \mathrm{~T}$ at the Institute of Advanced Biomedical Technologies, Chieti, Italy. An initial T1-weighted anatomical (3-D MP-RAGE pulse sequence; $1 \mathrm{~mm}$ isotropic voxels) and $\mathrm{T} 2 *$-weighted functional data were collected with an eightchannel phased-array head coil. EPI data (gradient-echo 
pulse sequence) were acquired from 31 slices $(3.5 \times$ $2.875 \times 2.875 \mathrm{~mm}$ resolution, repetition time $=2000 \mathrm{msec}$, echo time $=64 \mathrm{msec}$, SENSE factor $=2$, flip angle $=$ $80^{\circ}$, field of view $=230 \mathrm{~mm}$ ). Slices were oriented parallel to the AC-PC axis of the observer's brain.

\section{Experimental Procedure and Materials}

The participant was in a supine position in the fMRI scanner for about $1 \mathrm{hr}$ and completed seven fMRI runs. A wooden table was placed on the participant's legs. The participant's right hand was placed at the center of the table on an object (brush for body massage). A fake hand (mannequin) and the hand of another individual (another volunteer who was standing next to the scanner) were both placed next to the participant's hand. To keep the participants naive about whose hand was placed on the table, they were not introduced to the other person before the experiment and it was not possible for them to see the hand or the individual they were touching during the experiment. To avoid systematic effects of the location where the human and fake hand were placed, their position was pseudorandomized throughout the experiment (i.e., on the right and left side of the participant's hand). Before each individual fMRI run, the participant was informed about on which side of his or her own hand the human and fake hands were placed. Behavioral performance accuracy of participants was monitored during the experiment through a video camera placed in the MRI room and proved that all the participants were accurate in the performance of the task. The experimental setup is depicted in Figure 1.

During the touch intention fMRI runs (duration: 194 functional volumes $/$ run $=6.46 \mathrm{~min} / \mathrm{run}$ ), the participants completed a series of touch and no touch trials. Trial order was randomized. Each trial, either touch or no touch, started with a visual cue consisting of two black and white line outline drawings. The upper drawing indicated the modality of the touch (i.e., how the touch had to be performed), whereas the lower drawing indicated the target of the touch (what had to be touched). The modality could be either the participant's own hand or an object (brush for body massage). The target could either be the

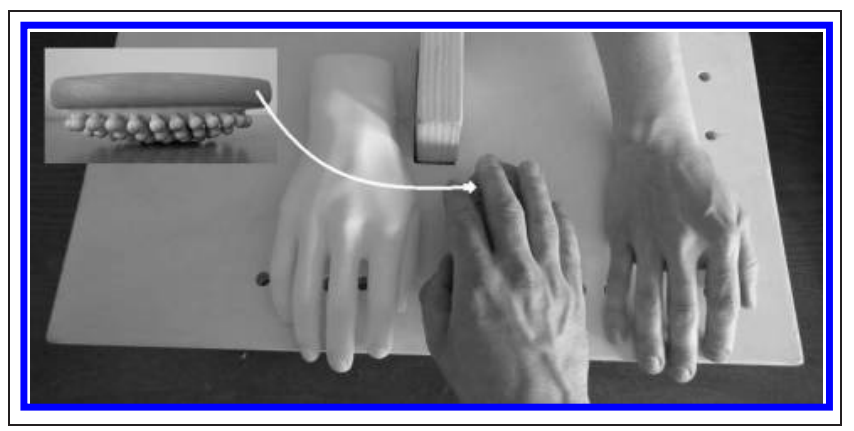

Figure 1. Picture of the experimental setup. human or the fake hand. Thus, four types of cues could be distinguished: "hand/human hand," "hand/fake hand," "object/human hand," and "object/fake hand." The experimental procedure of the touch intention runs is illustrated in Figure 2.

The visual cues were presented for a duration of $1000 \mathrm{msec}$ and were always followed by a red fixation cross. After $3000 \mathrm{msec}$, the red fixation cross could become either blue (duration $=6000 \mathrm{msec}$ ) or black (variable duration $=2000 / 4000 / 6000 / 8000 \mathrm{msec}$ ). When the red fixation cross became blue (20\% of the trials), the participants were required to perform a gentle massage of the dorsum of the human hand or of the fake hand in the same manner. These trials were defined as "touch trials" and mainly served as catch trials in the experiment. According to the cue, participants had to massage the human hand with their own hand (14 trials), the human hand with the object (14 trials), the fake hand with their own hand (14 trials), or the fake hand with the object (14 trials). When the blue fixation cross turned black, participants had to bring their hand back to the original position on the table. We chose to massage the dorsum of the hand for two reasons: (1) to extend previous studies where we showed that the mere social perception of the dorsum of another individual's hand being touched activated somatosensory cortices (Ebisch et al., 2008, 2011) and (2) the dorsum of the hand has been related to clear social functions, like affiliative social body contact, mainly based on the presence of C-tactile afferents (Morrison et al., 2010; Löken, Wessberg, Morrison, McGlone, \& Olausson, 2009).

In case the red fixation cross became black (80\% of the trials), participants had to keep their hand on the table and to wait for the next cue. These trials were defined as "no touch trials." Because the touch trials occurred randomly, participants could not know beforehand whether they had to perform the cued touch and were required to prepare the cued touch in all the trials (i.e., "touch trials" as well as "no touch trials"). The "no touch trials" were of principal interest for data analysis, because they reflected the intention to touch, without the presence of any overt movements of the participant. Thus, the touch intention could be "hand/human hand" (56 trials), "object/human hand" (56 trials), "hand/fake hand" (56 trials), or "object/ fake hand" (56 trials).

In addition to these touch intention fMRI runs, all participants underwent a tactile localizer task always run at the end of the fMRI session during which they were touched (i.e., gentle massage) on their right hand in randomized order by the hand of the individual standing next to the scanner $(8 \times 10 \mathrm{sec}$ periods $)$ or by the brush for body massage $(8 \times 10 \mathrm{sec}$ periods $)$. Touch periods were divided by an intertrial interval of $12 \mathrm{sec}$. Participants were touched on the dorsum of their hand to match the passive touch experience condition (i.e., being touched on the dorsum of one's own hand) with the active touch conditions where the other individual passively 
Figure 2. (A) Cue stimuli for the experimental conditions. (B) Time line of the experimental paradigm including touch as well as no touch trials. During the "cue" phase, one of the cues depicted in (A) is presented according to a randomized order.

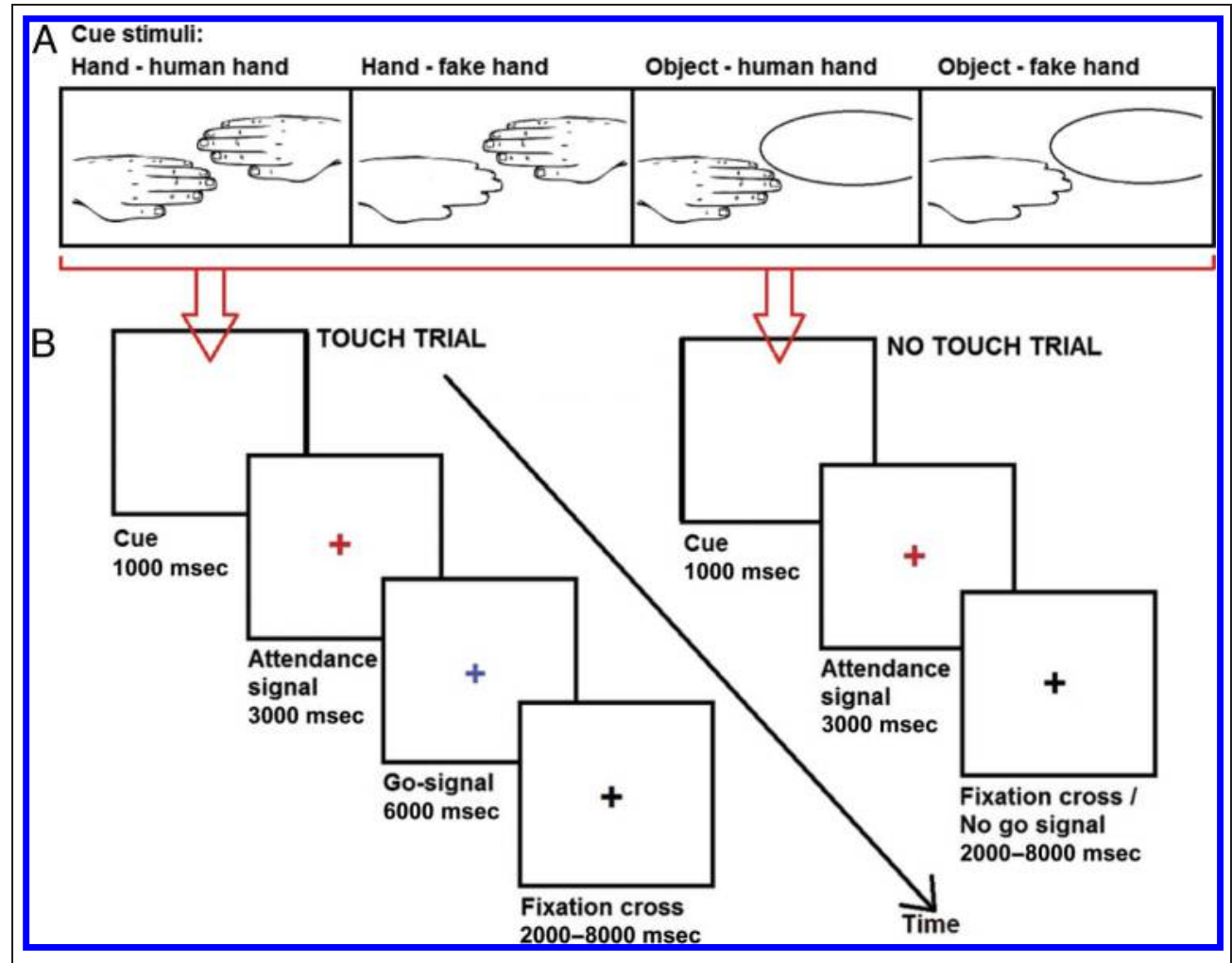

experienced touch in the same way. The localizer task was separated from the active touch conditions, allowing the use of an independent data set for creating a tactile localizer mask, thus providing an independent way to select voxels responding to passive tactile stimulation. Analyzing modulation of BOLD response in anticipation of active touch condition in brain regions involved in passive touch experiences was of theoretical interest for the study, whereas the relationship between active and passive touch was not to be investigated directly.

Before scanning, participants underwent a practicing session outside the scanner to train them on the fMRI task with the experimenter, thus avoiding interactions with the individual who was going to stay next to the scanner during the experiment. At debriefing, participants were asked to rate the pleasantness of their experience of actively touching the human hand or the fake hand by means of their own hand or the object. This rating referred to the pleasantness of the active touch as required by the experimental design during scanning. For this purpose, the pleasantness of the four different touch stimulations was rated on a visual analog scale.

\section{fMRI Data Preprocessing and Analysis}

Raw data were analyzed with Brain Voyager QX 2.3 software (Brain Innovation, Maastricht, The Netherlands). Because of T1 saturation effects, the first five scans of each run were discarded from the analysis. Preprocessing of functional data included slice scan time correction, motion correction, and removal of linear trends from voxel time series. A 3-D motion correction was performed with a rigid body transformation to match each functional volume to the reference volume estimating three translation and three rotation parameters. Preprocessed functional volumes of a participant were coregistered with the corresponding structural data set. As the 2-D functional and 3-D structural measurements were acquired in the same session, the coregistration transformation was determined using the slice position parameters of the functional images and the position parameters of the structural volume. Structural and functional volumes were transformed into the Talairach space (Talairach \& Tournoux, 1988) using a piecewise affine and continuous transformation. Functional volumes were resampled at a voxel size of $3 \times 3 \times 3 \mathrm{~mm}$ and spatially smoothed with a Gaussian kernel of $6 \mathrm{~mm}$ FWHM to account for intersubject variability.

The touch intention fMRI runs were modeled by means of a two gamma hemodynamic response function using predictors for the different no touch conditions (one regressor including cue and red cross representing the touch anticipation phase) and the different touch conditions (one regressor including cue and red cross representing the touch anticipation phase and one regressor for the blue cross representing the touch performance phase). The intertrial interval (black cross) was defined as a baseline period (rest) and, hence, not modeled as a separate predictor. The tactile localizer fMRI run was also modeled by means of a two gamma hemodynamic 
response function. In this case, the different types of touch were defined as separate predictors and the intertrial interval served as a baseline.

Before statistical analysis, a percent signal change normalization of the time series from the different runs was performed. The parameters (beta values) estimated in individual participant analysis were entered in a secondlevel voxel-wise random effect group analysis to search for activated areas that were consistent for the whole group of participants. The $p$ value $(<.001$ uncorrected) of the statistical maps and an estimate of the spatial correlation of voxels were used as input in a Monte Carlo simulation (1000 simulations) to access the overall significance level and to determine a cluster size threshold $(k)$ to obtain a significance level that was cluster level-corrected for multiple comparisons (Forman et al., 1995). The coordinates of the voxel clusters showing statistically significant effects were compared with the Talairach atlas available in Brain Voyager QX software to label them in terms of anatomically defined regions and Brodmann's areas.

Statistical maps related to touch anticipation (cue and red cross phase of the "no touch trials") as well as touch performance (blue cross phase of the "touch trials") were calculated by means of voxel-wise, whole-brain $t$ tests (contrast: any condition vs. baseline; "hand/human hand" vs. baseline or "hand/fake hand" vs. baseline or "object/human hand" vs. baseline or "object/fake hand" vs. baseline).

To investigate whether there were statistically significant modulations of BOLD response because of a different target (human hand vs. fake hand), voxel-wise statistical contrasts based on the $t$ statistic were performed specifying condition effects and interactions between conditions by appropriately weighted linear contrasts on the "no touch trials." In particular, it was investigated whether there were statistically significant Target, Modality, or Target $\times$ Modality interaction effects. Contrasts of principal interest were the effect of Target (is the anticipation of touching a human hand different from touching a fake hand?) and the Target $\times$ Modality interaction effect (is the anticipation of actively touching a human hand, compared with a fake hand, by one's own hand functionally distinct from anticipating the active touch of a human hand with an object?).

The Target $\times$ Modality interaction contrasts investigating whether the anticipation of touching a human hand was associated with stronger neural activity, compared with the control condition (touching a fake hand), were [(hand/human hand $>$ hand/fake hand) - (object/ human hand $>$ object/fake hand)] and [(object/human hand $>$ object/fake hand) - (hand/human hand $>$ hand/ fake hand)]. Whereas the former contrast allowed to test for differential BOLD response specifically when the touch was performed with one's own hand, the latter contrast allowed to test for differential BOLD response specifically when the touch was performed with an object.

With respect to the Target effect, we investigated whether the human hand elicited a greater BOLD sig- nal compared with the fake hand target, independent of modality: [human hand $>$ fake hand]. Regarding the modality effect, we investigated whether there was a difference in BOLD response between a touch performed with one's own hand and a touch performed with an object, independent of target: [hand > object] or [hand $<$ object].

First, the contrasts described above were performed by means of a whole-brain, voxel-wise approach. Second, to focus on brain voxels that also responded to firstperson tactile experiences, these contrasts were performed within the mask obtained by the tactile localizer task (touch experience vs. baseline).

To control for differences in BOLD response because of differences in affective valence between the conditions, ROI-based control analyses on BOLD responses to the "no touch trials" were performed, taking into account the affective valence of the touch. Individual beta values were extracted from the ROIs showing a significant effect regarding the above-described statistical contrasts (Target or interaction effects) between the experimental "no touch" conditions. Beta values for each ROI were calculated from the average signal time course of the voxels included in each ROI. ANCOVAs were performed on each cluster with these beta values within the clusters as dependent variables and Experimental Condition as withinsubject factor to specifically investigate the effect of the covariate avoiding double dipping for effects because of the experimental conditions. The difference scores between the pleasantness ratings of the experimental conditions were set as covariate. Thus, for the contrast [hand/ human hand $>$ hand/fake hand], the difference score was calculated for the pleasantness ratings of the "hand/human hand" and the "hand/fake hand" conditions. For the contrast [object/human hand $>$ object/fake hand], the difference score was calculated for the pleasantness ratings of the "object/human hand" and the "object/fake hand" conditions.

An additional whole-brain analysis was performed on the "touch trials." It needs to be mentioned that this analysis is rather exploratory, also because of the small number of touch trials and variability in touch performance; touch trials primarily served as catch trials. In this additional analysis, the same voxel-wise contrasts as reported for the "no touch" trails were performed on the "touch trials," specifically analyzing the phase when the blue cross was present, that is, when participants were actually performing the touch, excluding the preceding cue and red cross phases that were modeled as a separate regressor.

Finally, whole-brain, voxel-wise conjunction analyses were performed to preliminarily test whether the brain regions involved in the anticipation of active interpersonal touch also responded differently to actual touch performance. A random effect analysis of the conjunction between two contrasts was based on the minimum statistic compared with the conjunction null (Nichols, Brett, 
Andersson, Wager, \& Poline, 2005). This method controls the false positive error for conjunction inference and tests for common activations by creating the intersection of statistical maps thresholded at a specific alpha rate. Also in this case, analysis of touch anticipation concerned the "no touch trials" (i.e., one regressor including both the cue and red cross phases), whereas the analysis of touch performance focused on the touch performance phase of the "touch trials" (i.e., regressor concerning the blue cross phases). Two conjunction contrasts were performed: (1) ["hand/human hand" vs. "hand/fake hand" no touch trials] $\cap$ ["hand/human hand" vs. "hand/fake hand" touch performance] and (2) ["object/human hand" vs. "object/ fake hand" no touch trials] $\cap$ ["object/human hand" vs. "object/fake hand" touch performance].

\section{RESULTS}

\section{Pleasantness Ratings}

Average pleasantness rating (minimum $=0$, maximum $=$ 10) and standard deviation was for the "hand/human hand" touch $6.19 \pm 2.12$, for the "hand/fake hand" touch $4.90 \pm 1.87$, for the "object/human hand" touch $5.44 \pm$ 1.48 , and for the "object/fake hand" touch $3.76 \pm 2.19$. ANOVA showed a significant main effect of Target, $F(1$, $14)=8.271, p<.01$. Average ratings suggest that touching a human hand was rated by participants as being slightly more pleasant than touching a fake hand. There neither was significant main effect of Modality nor a significant Target $\times$ Modality interaction effect $(p>.05)$.

\section{fMRI Data Analysis: Experimental Conditions versus Baseline}

Compared with baseline, significant activation was found for the "no touch trials" (any condition vs. baseline) in bilateral superior frontal gyrus (BA 6), SMA (BA 6), dorsal ACC (BA 32), ventral precentral gyrus (BA 6), lateral and medial posterior parietal cortex (BA 7, BA 19), supramarginal gyrus (BA 40), superior frontal gyrus (BA 10), anterior insula (BA 13), nucleus caudatus, thalamus, occipital cortex (BA 17, BA 18, BA 19), fusiform gyrus (BA 37), right cerebellum, and left hemisphere postcentral gyrus (PostCG; SI), aIPL (BA 40), medial parietal cortex (BA 5; $p<.01$ corrected; $t>4.07 ; k>10)$.

Compared with baseline, significant activation was found for the touch performance phase (blue cross) of the "touch trials" (any condition vs. baseline) in bilateral dorsal precentral gyrus (BA 4), superior frontal gyrus (BA 6), SMA (BA 6), posterior parietal cortex (BA 5, BA 7, BA 39, BA 40), nucleus caudatus, putamen, parietal operculum/aIPL (BA 40), thalamus, anterior $/ \mathrm{mid} /$ posterior insula (BA 13), cerebellum, cingulate cortex (BA 24, BA 31, BA 32), mid brain, left PostCG (BA 1, BA 2, BA 3), and right ventral precentral gyrus (BA 6) and IOT (BA 19, BA 37; $p<.01$ corrected; $t>4.07 ; k>10$ ).
The observed activation patterns concerning touch anticipation and touch performance are largely consistent with previous studies investigating similar phenomena (e.g., Lederman \& Klatzky, 2009; Carlsson et al., 2000).

The tactile localizer task, compared with baseline, induced significant activation in left PostCG (SI; BA 3, BA 1, BA 2), posterior parietal cortex (BA 5, BA 7), aIPL (BA 40), dorsal precentral gyrus (BA 4), ventral precentral gyrus (BA 6), mid cingulate cortex (BA 31), lOT (BA 37), right anterior insula (BA 13, BA 45), and bilateral SII (BA 40) and posterior insula (BA 13; $p<.01$ corrected; $t>3.29 ; k>10)$.

\section{Anticipating an Active Touch of a Human versus a Fake Hand: Whole-brain Approach}

The interaction contrast [(hand/human hand $>$ hand/ fake hand) - (object/human hand > object/fake hand)] showed a significant effect in left PostCG (SI; BA 2), right cerebellum, and left medial pFC (MPFC; $p<.01$ corrected; $t>3.73 ; k>7$ ). The interaction effect in right cerebellum was driven by a stronger BOLD response in anticipation of a "hand/human hand" touch, compared with a "hand/fake hand" touch. The interaction effects in left PostCG (SI; BA 2) and left MPFC (BA 10, BA 32) were driven by stronger activity (i.e., weaker deactivation) in anticipation of a "hand/human hand" touch, compared with a "hand/fake hand" touch.

The contrast [(object/human hand $>$ object/fake hand $)-$ (hand/human hand $>$ hand/fake hand)] yielded significant clusters in left aIPL extending into postcentral sulcus (PostCS; BA 40/BA 2), and in left IOT (BA 37; $p<.01$ corrected; $t>3.73 ; k>5$ ). The interaction effects in left aIPL/PostCS and IOT were driven by stronger activity during the "object/human hand" condition, compared with the "object/fake hand" condition.

No significant positive effect was detected for the human hand target, compared with the fake hand target, independent of modality. Significant effects for modality, independent of target, were found in left precentral gyrus, left PostCG, left mid insula, right PostCG, bilateral PreCG, and left mid cingulate cortex, reflecting stronger BOLD responses when anticipating a touch with the object, compared with a touch with one's own hand $(p<.01$ corrected; $t>3.73 ; k>7)$, and in left occipital cortex reflecting stronger BOLD responses when anticipating a touch with one's own hand, compared with a touch with the object $(p<.01$ corrected; $t>3.73$; $k>7)$.

Group statistical maps with voxel clusters showing a significant interaction effect at the whole-brain level and graphs representing average percent signal change within these voxel clusters are depicted in yellow/green in Figure 3 for the intention to touch with one's own hand and in Figure 4 for the intention to touch with the object. Further details about these clusters and statistical information are reported in Table 1. 
ROI-based covariance analysis failed to detect a significant effect of pleasantness ratings as covariate in these ROIs showing an interaction effect $(p>.1$ corrected for multiple comparisons, that is, for the number of ROIs included in the analysis), even using an uncorrected threshold of $p<.1$. Thus, although the different types of intended touch required by the experimental paradigm slightly differed regarding their pleasantness as experienced by the individual participants, the distinct activation patterns could not be explained by the pleasantness of the touch the participants intended to perform.

\section{Anticipating an Active Touch of a Human versus a Fake Hand: Brain Regions Responding to Tactile Stimulation}

Voxel-wise contrasts regarding the "no touch trials" within the tactile localizer mask showed that the significant inter- action effect for the contrast [(hand/human hand $>$ hand/ fake hand) - (object/human hand > object/fake hand)] inleft PostCG (SI; BA 2) concerned voxels that also responded to passive tactile experiences $(p<.01$ corrected; $t>3.73 ; k>5$ ). Right cerebellum and left MPFC did not respond to passive touch experiences.

The contrast [ (object/human hand > object/fake hand) - (hand/human hand $>$ hand/fake hand)] yielded significant clusters in left aIPL extending into PostCS (BA 40/BA 2) and in left lOT (BA 37; $p<.01$ corrected; $t>3.73 ; k>5$ ) indicating that these regions also responded to passive tactile experiences.

No significant positive effect was detected for the human hand target, compared with the fake hand target, independent of modality. A significant effect for modality, independent of target, was found in left precentral gyrus, left PostCG, left mid insula, reflecting stronger BOLD responses when anticipating a touch with the

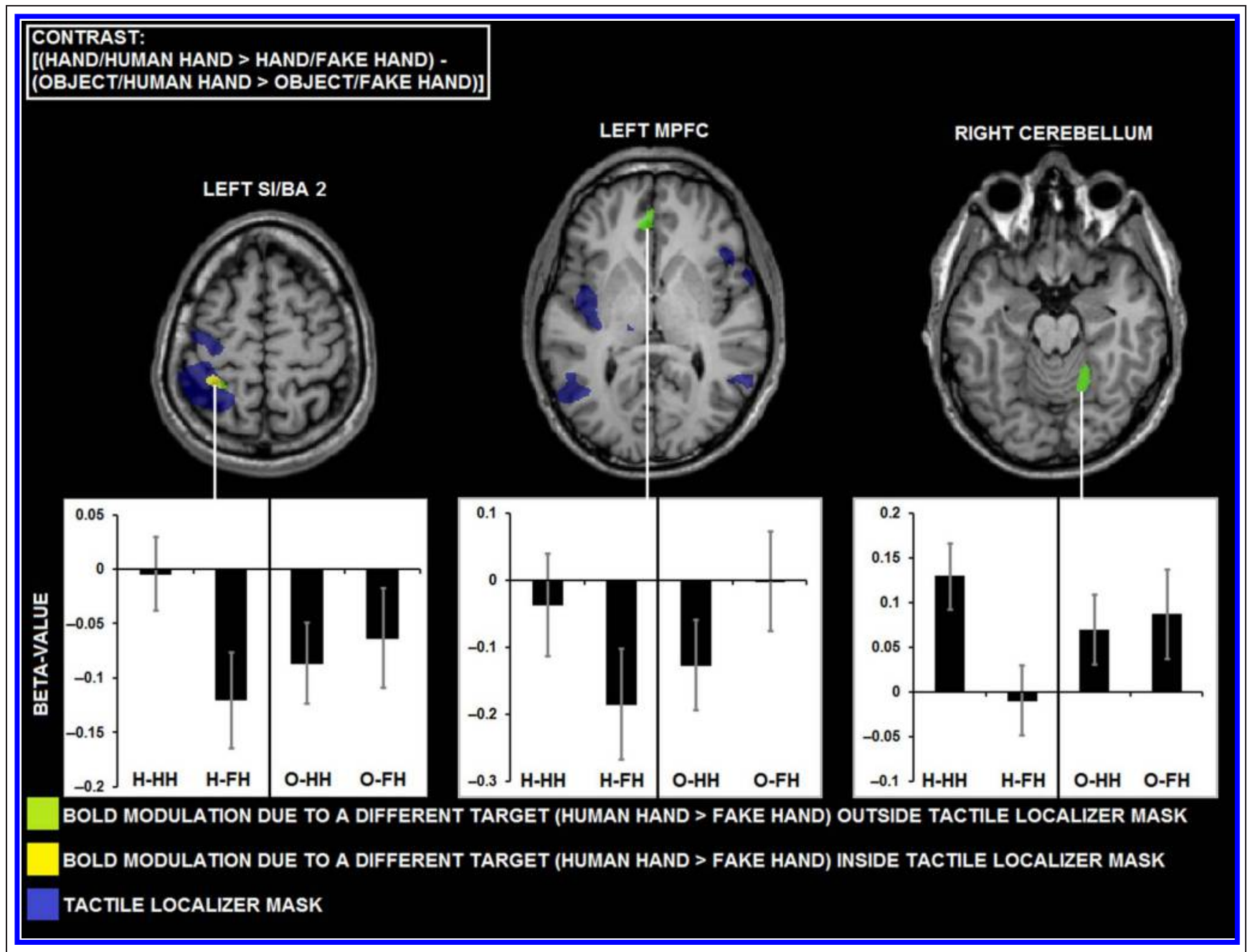

Figure 3. Group statistical maps of voxel clusters (yellow = inside tactile localizer mask; green = outside tactile localizer mask) showing a significant modulation in BOLD response because of the different touch target (human hand $>$ fake hand), when intending to touch with one's own hand ( $p<.01$, corrected). The tactile localizer mask is depicted in blue. Graphs represent average percent signal change, compared with baseline, in the voxel clusters and standard errors for the different no touch conditions. H-HH = hand/human hand; H-FH = hand/fake hand; O-HH = object/human hand; O-FH = object/fake hand. 
Figure 4. Group statistical maps of voxel clusters (yellow $=$ inside tactile localizer mask; green $=$ outside tactile localizer mask) showing a significant modulation in BOLD response because of the different touch target (human hand $>$ fake hand), when intending to touch with the object $(p<.01$, corrected). The tactile localizer mask is depicted in blue. Graphs represent average percent signal change, compared with baseline, in the voxel clusters and standard errors for the different no touch conditions. $\mathrm{H}-\mathrm{HH}=$ hand/human hand; $\mathrm{H}-\mathrm{FH}=$ hand/fake hand; $\mathrm{O}-\mathrm{HH}=$ object/human hand; O-FH = object/fake hand.

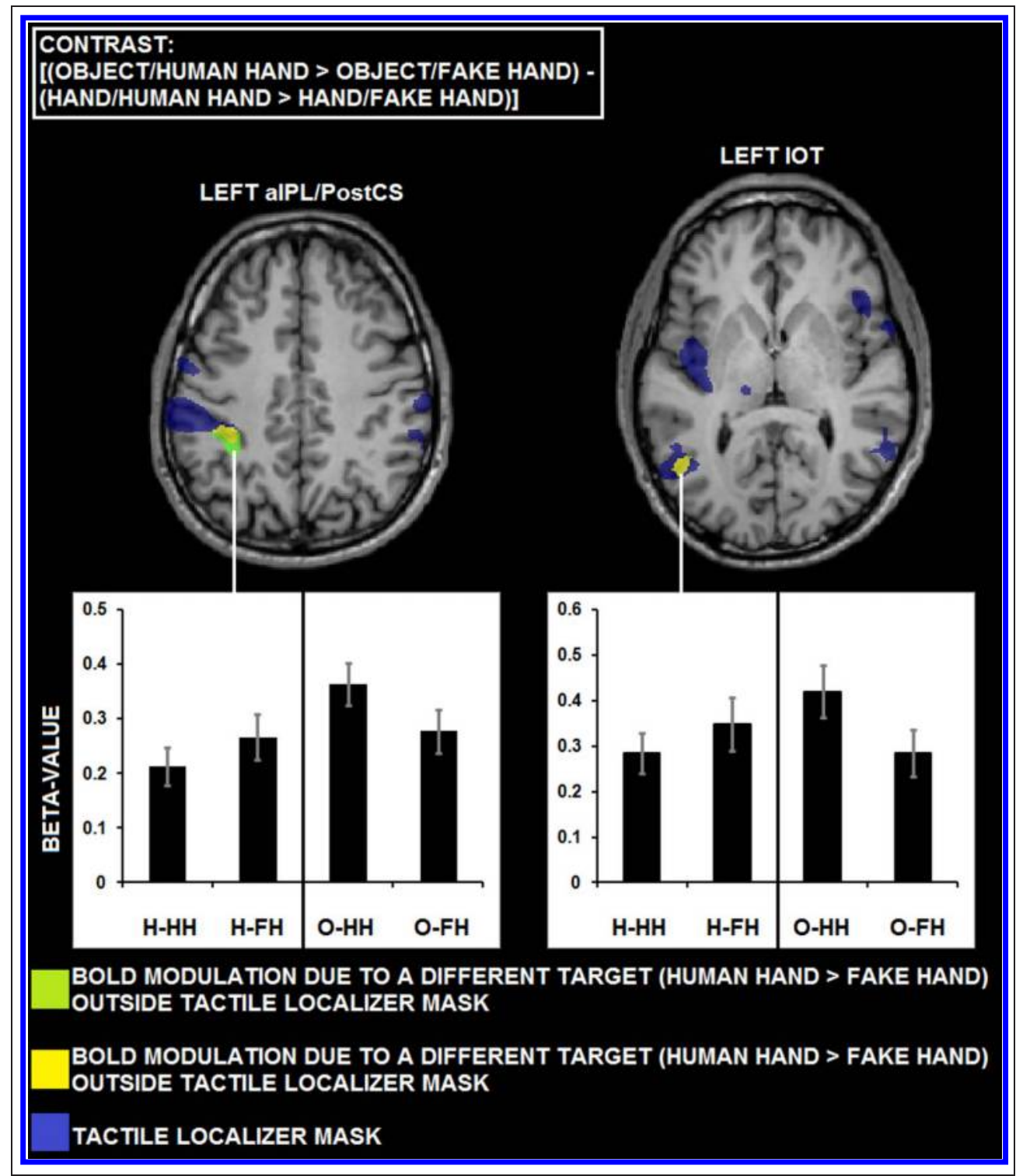

object, compared with a touch with one's own hand $(p<.01$ corrected; $t>3.73 ; k>5)$. The opposite modality contrast [hand $>$ object] did not yield significant results within the tactile localizer mask.

Group statistical maps with voxel clusters showing a significant interaction effect inside the tactile localizer mask and graphs representing average percent signal change within these voxel clusters for the different conditions are depicted in yellow in Figure 3 for the intention to touch with one's own hand and in Figure 4 for the intention to touch with the object. Further details about these clusters and statistical information are reported in Table 1.

ROI-based covariance analysis failed to detect a significant effect of pleasantness ratings as covariate in these ROIs showing a significant interaction effect $(p>.1$ corrected for multiple comparisons, i.e., for the number of ROIs included in the analysis), even using an uncorrected threshold of $p<.1$. Hence, the detected differences in brain activity inside the tactile localizer mask seem to reflect sensorimotor processes rather than the affective aspect of active interpersonal touch.

\section{Touch Performance}

An exploratory analysis concerning the touch trials based on whole-brain, voxel-wise contrasts showed a significant interaction effect for the contrast [(hand/human hand $>$ hand/fake hand) - (object/human hand $>$ object/fake hand)] in left MPFC, left precuneus, right putamen, right medial-temporal cortex, and right 1OT $(p<.01$ corrected; $t>4.07 ; k>7$ ), reflecting stronger activity during the "hand/human hand" condition, compared with the "hand/fake hand" condition.

For the interaction contrast [(object/human hand $>$ object/fake hand) - (hand/human hand $>$ hand/fake hand)], a significant effect was found in left PostCG 
and right posterior parietal cortex $(p<.01$ corrected; $t>4.07 ; k>7$ ), reflecting stronger activity during the "object/human hand" condition, compared with the "object/fake hand" condition.

A significant positive effect was detected for the human hand target, compared with the fake hand target, in MPFC, precuneus, and right posterior STS $(p<.01$ corrected; $t>4.07 ; k>7$ ) reflecting increased activity for the human hand target, compared with the fake hand target, independent of modality. A significant effect of modality was found in bilateral precentral gyrus, bilateral PostCG, bilateral SMA, bilateral posterior parietal cortex, bilateral SII, bilateral posterior insula, bilateral precuneus, left MPFC, right putamen, right posterior STS, bilateral cerebellum $(p<.01$ corrected; $t>4.07 ; k>7)$, reflecting increased activity when touching with one's own hand, compared with an object, independent of the target.

\section{Conjunction Analysis: Touch Anticipation and Touch Performance}

Conjunction analysis showed that left MPFC was characterized by stronger activity during both the anticipation (i.e., weaker deactivation, compared with baseline) and the performance (i.e., stronger activation, compared with baseline) of the "hand/human hand" touch, compared with the "hand/fake hand" touch $(p<.01$ corrected, cluster size: 432 voxels, Talairach coordinates: $-7,46,21)$. By contrast, left PostCG (SI) showed stronger activity (i.e., weaker deactivation, compared with baseline) anticipating

Table 1. Brain Regions Showing a Modulation of BOLD Response by the Different Experimental Conditions and Statistical Information for the Direct Contrasts between the Touch Intention Conditions

\begin{tabular}{|c|c|c|c|c|c|}
\hline Brain Region & Brodmann's Area & $\begin{array}{l}\text { Peak Coordinates } \\
\quad(x, y, z)\end{array}$ & Cluster Size & $t$ & $p$ \\
\hline \multicolumn{6}{|l|}{ Inside Tactile Mask } \\
\hline \multicolumn{6}{|c|}{$\begin{array}{l}\text { Interaction contrast (corresponding to Figure 3, yellow): [(hand/human hand }>\text { hand/fake hand) - } \\
\quad(\text { object/human hand }>\text { object/fake hand)] }\end{array}$} \\
\hline LH PostCG (SI) & 2 & $-31,-41,57$ & 324 & 5.332 & $<.0001$ \\
\hline \multicolumn{6}{|c|}{$\begin{array}{l}\text { Interaction contrast (corresponding to Figure 4, yellow): [(object/human hand }>\text { object/fake hand) - } \\
\quad \text { (hand/human hand }>\text { hand/fake hand)] }\end{array}$} \\
\hline LH aIPL & 40 & $-43,-32,33$ & 567 & 4.607 & $<.0005$ \\
\hline LH IOT & 6 & $-49,-65,3$ & 243 & 4.926 & $<.0001$ \\
\hline \multicolumn{6}{|c|}{ Target effect [human hand $>$ fake hand] - Modality effect: [object $>$ hand] } \\
\hline LH PreCG & 6 & $-58,-5,30$ & 1269 & 7.098 & $<.00001$ \\
\hline LH PostCG/aIPL & $2 / 40$ & $-37,-35,42$ & 1242 & 5.094 & $<.0001$ \\
\hline LH mIC & 13 & $-46,-8,15$ & 1863 & 6.322 & $<.00001$ \\
\hline
\end{tabular}

Outside Tactile Mask

Interaction contrast (corresponding to Figure 3, yellow/green): [(hand/human hand $>$ hand/fake hand) (object/human hand $>$ object/fake hand)]

$\begin{array}{lccccc}\text { RH Cerebellum } & - & 17,-56,-18 & 243 & 4.157 & <.001 \\ \text { LH MPFC } & 10 / 32 & -7,40,3 & 162 & 3.921<.001\end{array}$

Interaction contrast: [(object/human hand > object/fake hand) - (hand/human hand $>$ hand/fake hand) $]-$

Target effect [human hand $>$ fake hand] - Modality effect: [hand $>$ object]

$\begin{array}{lllll}\text { LH OCC } & 18 & -10,-92,-12 & 8343 & 9.036\end{array}$

Modality effect: [object $>$ hand]

\begin{tabular}{|c|c|c|c|c|c|}
\hline RH PreCG & 4 & $32,-26,60$ & 6075 & 8.471 & $<.00001$ \\
\hline RH PostCG & 2 & $41,-29,42$ & - & 7.844 & $<.00001$ \\
\hline LH PreCG & 4 & $-37,-26,51$ & 13698 & 6.398 & $<.00005$ \\
\hline LH MCC & 31 & $-10,-14,45$ & 351 & 5.746 & $<.00005$ \\
\hline
\end{tabular}

$\mathrm{LH}=$ left hemisphere; $\mathrm{RH}=$ right hemisphere; PreCG = precentral gyrus; MFG = middle frontal gyrus; PPC = posterior parietal cortex; OCC $=$ occipital cortex; $\mathrm{MCC}=$ mid cingulate gyrus; $\mathrm{mIC}=$ mid insular cortex. Peak coordinates refer to Talairach space. 
Figure 5. Group statistical maps of voxel clusters showing a significant modulation in BOLD response by target (human hand or fake hand) both during the anticipation and the performance of the touch when performed with one's own hand $(p<.01$, corrected). Graphs represent average percent signal change, compared with baseline, in the voxel clusters and standard errors. $\mathrm{H}-\mathrm{HH}=$ hand/human hand; $\mathrm{H}-\mathrm{FH}=$ hand/fake hand.

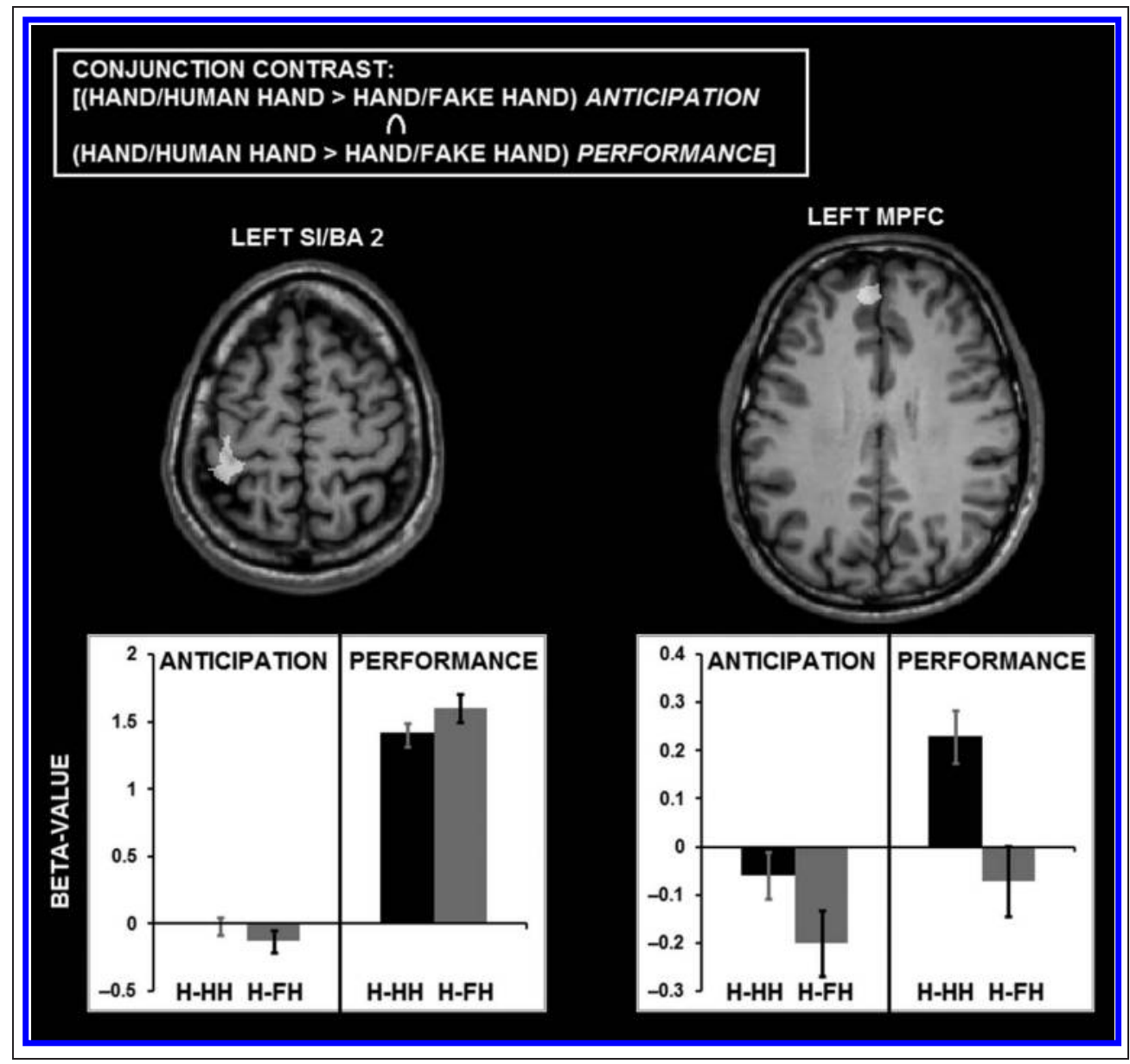

a "hand/human hand" touch, compared with a "hand/fake hand" touch, whereas neural activity was stronger (i.e., stronger activation, compared with baseline) during the subsequent performance of a "hand/fake hand" touch, compared with a "hand/human hand" touch $(p<.01$ corrected, cluster size: 2457 voxels, Talairach coordinates: $-40,-41,57)$. Concerning the touch by an object, conjunction analysis did not detect voxel clusters characterized by a significant modulation by target during both touch anticipation and performance. Group statistical maps with voxel clusters showing a significant conjunction effect and graphs representing average percent signal change within these voxel clusters for the anticipation of touch (i.e., "no touch trials") and the performance of touch (i.e., touch phase of the "touch trials") are depicted in Figure 5.

\section{DISCUSSION}

This study aimed at investigating the anticipatory neural representations of active interpersonal touch. It was hypothesized that the intention to touch another individual (i.e., animate target, human hand) would be accompanied by differential neural activity in somatosensory cortices when compared with the control condi- tion, that is, the intention to touch an inanimate target (i.e., fake hand). Confirming this hypothesis, fMRI results yielded stronger neural activity anticipating the touch of a human hand, compared with the touch of a fake hand in left PostCG (SI/BA 2), MPFC, aIPL, and IOT and right cerebellum. A tactile localizer task showed that these regions, except for left MPFC and right cerebellum, were also endowed with somatosensory properties underlying the first-person experience of touch.

No action performance (i.e., touching) was required in the conditions included in the statistical analysis of anticipatory processes and no overt hand movements were observed. Therefore, these results cannot be attributed to action performance or differences in tactile experiences between touching a human hand or a fake hand per se. The only difference between the conditions was the intention to eventually touch either an animate or an inanimate target controlling for nonsocial anticipatory sensorimotor processes by using a fake hand as inanimate target. Analysis of touch performance did not show significant target or Target $\times$ Modality effects in (pre)motor cortices, further suggesting that the anticipated touch conditions were comparable in motor performance. Finally, the participants were kept unaware of the identity of the individual they had to touch to control for possible confounds because 
of interpersonal relationships, whereas covariance analysis showed that the observed effects were independent from the affective valence of the touch as experienced by the participants. Thus, the differential modulation of activation induced by the no touch conditions is proposed to reflect sensorimotor processes specifically distinguishing between the anticipation of active animate or inanimate touch.

Importantly, interpersonal touch was studied in two modalities. Participants were cued to touch another individual's hand by means of their own hand or by using an object. As expected, a significant Target $\times$ Modality interaction effect in the brain regions described above showed that these conditions, compared with the conditions in which participants were cued to touch a fake hand, induced differential activation patterns. Below, we will further elaborate on this.

\section{Anticipating Active Skin-to-Skin Contact}

Differential neural activity in anticipation of skin-to-skin contact, that is, the touch of a human hand, compared with a fake hand, was detected in SI/BA 2. This finding extends previous work by showing that SI also may participate in active touch by specific anticipatory processes depending on the target of the touch. In particular, a more detailed examination of the fMRI results reveals that the active touch of a fake hand with one's own hand is anticipated by a stronger deactivation in SI, that is, a suppression of the BOLD signal, compared with baseline (Schäfer et al., 2012; Devor, Tian, Nishimura, Teng, \& Hillman, 2007; Hlushchuk \& Hari, 2006; Shmuel, Augath, Oeltermann, \& Logothetis, 2006). Such a deactivation was absent in anticipation of the active touch of a human hand. We propose that such an anticipation could be based on sensory prediction mechanisms guiding behavior.

Sensory prediction or anticipation has a crucial role in motor control (Shadmehr, Smith, \& Krakauer, 2010; Wolpert \& Flanagan, 2001). According to forward models, an efference copy of the motor command is used to predict the sensory consequence of an action (Bays, Flanagan, \& Wolpert, 2006; Blakemore, Wolpert, \& Frith, 1999). Such prediction may lead to sensory attenuation or amplification in terms both of their phenomenology and of their cortical response (Hughes, Desantis, \& Waszak, 2013; Jackson et al., 2011; Voss et al., 2008; Blakemore, Wolpert, \& Frith, 1998; Chapman, 1994).

No studies directly compared the anticipatory processes related to the active touch of an animate and inanimate target. However, in the case of passively experienced touch, social factors have been demonstrated to modulate somatosensory responses as well as tactile perception (Gordon et al., 2013; Gazzola et al., 2012). Furthermore, Gazzola et al. (2012) showed by means of multivariate cluster analysis that neural activity in SI already differentiated between the type of touch the participants thought and predicted to receive before actually being touched. The present results add that actively touching an animate or an inanimate target is anticipated by differential activation patterns in bilateral SI/BA 2. Given the somatosensory and social functions of SI/BA 2 in combination with its connections with cortical motor circuits (Keysers et al., 2010), these findings may open a new window into the investigation of their contribution to social motor behavior.

Except for SI, increased anticipatory BOLD response was found for active skin-to-skin contact in right cerebellum. Not coincidentally, the cerebellum is a crucial brain structure underlying sensory prediction and the encoding of prediction errors (Roth, Synofzik, \& Lindner, 2013; Schlerf, Ivry, \& Diedrichsen, 2012; Blakemore \& Sirigu, 2003; Wolpert, Miall, \& Kawato, 1998). It has been generally suggested that the cerebellum is needed to optimize action performance and perception by recalibrating predictions of the sensory consequences of actions (Bastian, 2006; Wolpert et al., 1998). In particular, cerebellum could be involved in altering the perception of the effect of one's action by providing predictions about the sensory consequences of motor commands (Hughes et al., 2013; Blakemore, Frith, \& Wolpert, 2000; Wolpert et al., 1998). The effects reported here in both SI and cerebellum are in line with previous evidence suggesting that sensory prediction in the cerebellum might modulate activity in somatosensory cortices when tactile stimuli are selfgenerated (Blakemore et al., 1999).

What could this sensory prediction entail? A relevant finding is that the absence of a deactivation in BA 2/SI and the presence of a positive anticipatory response in cerebellum were observed specifically when the participants prepared to touch the human hand directly with their own hand, that is, when the cue implied an eventual bodily contact with the target. According to previous reports on cerebellar function and sensory prediction, it has been proposed that the comparison of the predicted signals with actual sensory feedback facilitates the distinction between the sensory consequences of one's own movements and externally produced sensory signals, thus augmenting sensitivity to external sensory cues during active touch (Bays et al., 2006; Blakemore et al., 1999).

It could be speculated that actively touching someone else's hand with one's own hand is linked with a greater sensitivity to errors or to externally produced sensory cues. Preliminary results obtained by conjunction analysis indicating increased activation in left SI/BA 2 during the actual performance of the "hand/fake hand" touch, compared with the "hand/human hand" touch, seems to support this hypothesis. Stronger anticipatory activity (i.e., weaker deactivation) for the "hand/human hand" touch may be followed by attenuated somatosensory activity during actual performance which in turn is associated with a greater tactile discrimination capacity (Hughes et al., 2013; Bays et al., 2006; Blakemore et al., 1999). An intriguing issue for future studies would be to investigate whether the mere intention to perform an interpersonal 
touch is sufficient to modulate perceptual sensitivity and how this may regulate motor control. In addition, further experiments will need to extricate the relevance of sensory feedback when comparing the active touch of animate and inanimate targets.

Additional stronger activity during the anticipation of skin-to-skin contact was found in left MPFC. Like in SI/ BA 2, a more detailed examination of the fMRI results discloses that the active touch of a fake hand with one's own hand is anticipated by a stronger deactivation in MPFC, that is, a suppression of the BOLD signal, compared with baseline. Conjunction analysis further yielded a significant modulation of BOLD response during both the anticipation and the subsequent performance of a hand touch in left MPFC: Whereas MPFC showed a stronger deactivation (negative BOLD modulation) in anticipation of a "hand/fake hand" touch, compared with the anticipation of a "hand/human hand" touch, MPFC showed enhanced BOLD response to the performance of a "hand/human hand" touch, compared with a "hand/ fake hand touch." MPFC has been associated with cognitive aspects of social cognition, like mentalizing (Amodio \& Frith, 2006; Gilbert et al., 2006), suggesting that during active skin-to-skin contact processes related to social reasoning also come into play, consistent with previous studies on social interaction (Schippers, Roebroeck, Renken, Nanetti, \& Keysers, 2010; Gilbert et al., 2007). We propose that the stronger deactivation in MPFC for the "hand/fake hand" no touch condition could reflect an anticipatory suppression of social reasoning processes that would be relatively irrelevant during the subsequent touch of the fake hand.

\section{Active Interpersonal Touch Mediated by an Object}

By contrast, in anticipation of an active interpersonal touch mediated by an object ("object/human hand" condition, compared with the "object/fake hand" control condition), a different set of brain regions was activated. There are some principal differences with the "hand/human hand" condition. The "object/human hand" condition required the use of an object not allowing to perceive distinct tactile properties of either the human or the fake hand when touch performance was required. Whereas the "object/fake hand" condition controlled for sensorimotor processes related to object manipulation, the absence of direct bodily contact with the target allowed to control for confounds because of differences in the predicted sensations associated with the animate and inanimate target. Thus, we propose that increased anticipatory activation in left aIPL/PostCS and 1OT for the active touch of a human hand with an object is associated with the intention to induce tactile sensations in another individual.

The aIPL activation cluster is likely located in area PF (Caspers et al., 2008). Cytoarchitectonic, functional, and anatomical studies suggest that the rostral aspect of aIPL represents the putative human homologue of monkey area PF (Caspers et al., 2006), with similar motor (Binkofski et al., 1999), somatosensory (Ruben et al., 2001), and social functions (Caspers, Zilles, Laird, \& Eickhoff, 2010; Gazzola \& Keysers, 2009; Buccino et al., 2001) as well as connectivity patterns (Wang et al., 2012). Studies in macaque monkeys show that area PF contains neurons with somatosensory and proprioceptive responses that also discharge in association with movements, especially goal-directed motor acts (Gallese et al., 2002; Hyvärinen, 1982). In particular, area PFG represents motor acts related to the hand and also responds to tactile input from the same hand and to proprioceptive input induced by arm flexion (Rozzi, Ferrari, Bonini, Rizzolatti, \& Fogassi, 2008). Consistent with these sensorimotor properties, PFG is connected with the hand representation of SII-PV complex, and with ventral (F5 and F4) and dorsal (F2) premotor areas (Rozzi et al., 2006; Petrides \& Pandya, 1984). Additionally, based on the detection of mirror neurons in area PFG (Fogassi et al., 2005; Gallese et al., 2002), PFG has been proposed to play a role in others' intention understanding (Bonini et al., 2010; Rizzolatti \& Sinigaglia, 2010). Most relevant to our study, showing aIPL involvement in anticipation of an action with a specific goal, is that PFG neurons have been shown to reflect the final goal of a performed action from the early phase of action unfolding (Bonini et al., 2011).

In addition to the mirror properties related to action performance and observation in aIPL, some studies suggested a more general role of aIPL in social perception, also including the mapping of others' tactile experiences onto one's own sensory representations. For example, in a previous study, we found that aIPL not only activates in response to tactile input, but also for the mere observation of another individual being touched (Ebisch et al., 2008). Furthermore, Morrison et al. (2013) postulated that aIPL, together with PostCG, may subserve the anticipation of the sensory consequences of observed hand-object interactions through the integration of action information and external information about the object. Here, we extend these findings by showing that aIPL and PostCS also activate in anticipation of active interpersonal touch without the need for any sensory input. Like in the case of social perception, this possibly incorporates the predicted sensory experiences of the person who is going to be touched by mapping them onto the somatosensory representation of our own tactile experiences (Keysers et al., 2010). A similar principle could apply to $1 \mathrm{OT}$, a multisensory region responding both to tactile stimulation (Hagen et al., 2002) and to the sight of touch (Ebisch et al., 2008). This is consistent with the proposal that multimodal circuits in the brain driving one's actions and sensations can ground an experience-based perception and generate expectations of others' sensations during social interaction based on embodied simulation (Morrison et al., 2013; Gallese \& Sinigaglia, 2011; Sebanz et al., 2006; Wilson \& Knoblich, 2005; Gallese, 2003; Gallese \& Goldman, 1998). 
Interestingly, it was proposed that the transformation of sensory information into a motor format is the main organization principle of aIPL (Rozzi et al., 2008). Moreover, PostCS as the posterior part of postcentral somatosensory corresponds to BA 2, a region projecting onto primary motor cortex (Caria et al., 1997; Kaneko et al., 1994a, 1994b), with a crucial role in motor control during haptic behavior (Freund, 2003; Iwamura \& Tanaka, 1996; Hikosaka et al., 1985). Hence, we argue that during actively touching another individual, using an object, others' predicted somatosensory experiences could be integrated with motor programs in aIPL/PostCS. Such an integration possibly supports action coordination when individuals interact (Sebanz et al., 2006), like the regulation of active interpersonal touch.

\section{General Discussion and Conclusions}

This study sheds new light on the neural bases of anticipatory processes of active interpersonal touch. Some additional issues need to be mentioned. Some caution may be required for the interpretation of the observed deactivations in SI/BA 2 and MPFC anticipating active touch, because relatively short intertrial intervals were used due to the rapid event-related fMRI paradigm and the experiment did not include long-lasting rest conditions in which no stimuli were applied.

Moreover, although we controlled for possible confounds because of interpersonal relationships or socioemotional valence, we should add that, in everyday life, in addition to the sensorimotor component, interpersonal touch is inherently associated with an affective component, too (Gallace \& Spence, 2010; Morrison et al., 2010). Motivational factors, like desire and aversion, probably will play a crucial role in the anticipation of interpersonal touch. Future work also will need to address the anticipatory processes representing the affective components of interpersonal touch and how these interact with sensorimotor predictions for the regulation of social behavior.

In conclusion, different from previous work on the contribution of the sensorimotor system to social cognition, mainly focused on social perception from a third-person perspective, the present findings provide new insights on the contribution of somatosensation, sensory prediction, and simulation mechanisms to social behavior in direct bodily interactions requiring to alternate between third- and second-person perspectives (see Gallese, in press; Gallese \& Ebisch, 2013). Moreover, we suggest that direct and indirect bodily tactile contact with conspecifics is approached with a different emphasis on personal perception and external goals, respectively.

\section{Acknowledgments}

This work was supported by the EU Grant TESIS (Towards an Embodied Science of InterSubjectivity) to V. G. The authors thank the two anonymous reviewers for their valuable comments on a previous version of this work.
Reprint requests should be sent to Sjoerd J. H. Ebisch, Institute of Advanced Biomedical Technologies, Department of Neuroscience and Imaging, G. d'Annunzio University, Via dei Vestini 33,66013 Chieti, Italy, or via e-mail: sjoerdebisch@yahoo.com or Vittorio Gallese, Department of Neuroscience, Section of Physiology, Parma University, Via Volturno 39, I-43100 Parma, Italy, or via e-mail: vittorio.gallese@unipr.it.

\section{REFERENCES}

Amodio, D. M., \& Frith, C. D. (2006). Meeting of minds: The medial frontal cortex and social cognition. Nature Reviews Neuroscience, 7, 268-277.

Bastian, A. J. (2006). Learning to predict the future: The cerebellum adapts feed forward movement control. Current Opinion in Neurobiology, 16, 645-649.

Bays, P. M., Flanagan, J. R., \& Wolpert, D. M. (2006). Attenuation of self-generated tactile sensations is predictive, not postdictive. PLoS Biology, 4, e28.

Binkofski, F., Buccino, G., Posse, S., Seitz, R. J., Rizzolatti, G., \& Freund, H. (1999). A fronto-parietal circuit for object manipulation in man: Evidence from an fMRI-study. European Iournal of Neuroscience, 11, 3276-3286.

Blakemore, S. J., \& Frith, C. D. (2003). Self-awareness and action. Current Otinion in Neurobiology, 13, 219-224.

Blakemore, S. J., Frith, C. D., \& Wolpert, D. M. (2000). The cerebellum is involved in predicting the sensory consequences of action. NeuroRebort, 12, 1879-1884.

Blakemore, S. J., \& Sirigu, A. (2003). Action prediction in the cerebellum and in the parietal lobe. Experimental Brain Research, 153, 239-245.

Blakemore, S. J., Wolpert, D. M., \& Frith, C. D. (1998). Central cancellation of self-produced tickle sensation. Nature Neuroscience, 1, 635-640.

Blakemore, S. J., Wolpert, D. M., \& Frith, C. D. (1999). The cerebellum contributes to somatosensory cortical activity during self-produced tactile stimulation. Neuroimage, 10 , 448-459.

Bonini, L., Rozzi, S., Serventi, F. U., Simone, L., Ferrari, P. F., \& Fogassi, L. (2010). Ventral premotor and inferior parietal cortices make distinct contribution to action organization and intention understanding. Cerebral Cortex, 20, 1372-1385.

Bonini, L., Serventi, F. U., Simone, L., Rozzi, S., Ferrari, P. F., \& Fogassi, L. (2011). Grasping neurons of monkey parietal and premotor cortices encode action goals at distinct levels of abstraction during complex action sequences. Iournal of Neuroscience, 31, 5876-5886.

Bosbach, S., Cole, J., Prinz, W., \& Knoblich, G. (2005). Inferring another's expectation from action: The role of peripheral sensation. Nature Neuroscience, 8, 1295-1297.

Bremmer, F., Schlack, A., Shah, N. J., Zafiris, O., Kubischik, M., Hoffmann, K., et al. (2001). Polymodal motion processing in posterior parietal and premotor cortex: A human fMRI study strongly implies equivalencies between humans and monkeys. Neuron, 29, 287-296.

Buccino, G., Binkofski, F., Fink, G. R., Fadiga, L., Fogassi, L., Gallese, V., et al. (2001). Action observation activates premotor and parietal areas in a somatotopic manner: An fMRI study. European Iournal of Neuroscience, 13, 400-404.

Caria, M. A., Kaneko, T., Kimura, A., \& Asanuma, H. (1997). Functional organization of the projection from area 2 to area 4gamma in the cat. Journal of Neurophysiology, 77, 3107-3114.

Carlsson, K., Petrovic, P., Skare, S., Petersson, K. M., \& Ingvar, M. (2000). Tickling expectations: Neural processing in anticipation of a sensory stimulus. Journal of Cognitive Neuroscience, 12, 691-703. 
Caspers, S., Eickhoff, S. B., Gever, S., Scheperjans, F., Mohlberg, H., Zilles, K., et al. (2008). The human inferior parietal lobule in stereotaxic space. Brain Structure $\mathcal{E}$ Function, 212, 481-495.

Caspers, S., Geyer, S., Schleicher, A., Mohlberg, H., Amunts, K., \& Zilles, K. (2006). The human inferior parietal cortex: Cytoarchitectonic parcellation and interindividual variability. Neuroimage, 33, 430-448.

Caspers, S., Zilles, K., Laird, A. R., \& Eickhoff, S. B. (2010). ALE meta-analysis of action observation and imitation in the human brain. Neuroimage, 50, 1148-1167.

Chapman, C. E. (1994). Active versus passive touch: Factors influencing the transmission of somatosensory signals to primary somatosensory cortex. Canadian Iournal of Physiology and Pharmacology, 72, 558-570.

Devor, A., Tian, P., Nishimura, N., Teng, I. C., \& Hillman, E. M. (2007). Suppressed neuronal activity and concurrent arteriolar vasoconstriction may explain negative blood oxygenation level-dependent signal. Journal of Neuroscience, 27, 4452-4459.

Dunbar, R. I. (2010). The social role of touch in humans and primates: Behavioural function and neurobiological mechanisms. Neuroscience and Biobehavioral Reviews 34, 260-268.

Ebisch, S. J., Ferri, F., Salone, A., Perrucci, M. G., D’Amico, L., Ferro, F. M., et al. (2011). Differential involvement of somatosensory and interoceptive cortices during the observation of affective touch. Iournal of Cognitive Neuroscience, 23, 1808-1822.

Ebisch, S. J., Perrucci, M. G., Ferretti, A., Del Gratta, C., Romani, G. L., \& Gallese, V. (2008). The sense of touch: Embodied simulation in a visuotactile mirroring mechanism for observed animate or inanimate touch. Iournal of Cognitive Neuroscience, 20, 1611-1623.

Fogassi, L., Ferrari, P. F., Gesierich, B., Rozzi, S., Chersi, F., \& Rizzolatti, G. (2005). Parietal lobe: From action organization to intention understanding. Science, 308, 662-667.

Forman, S. D., Cohen, J. D., Fitzgerald, M., Eddy, W. F., Mintun, M. A., \& Noll, D. C. (1995). Improved assessment of significant activation in functional magnetic resonance imaging (fMRI): Use of a cluster-size threshold. Magnetic Resonance in Medicine, 33, 636-647.

Freund, H. J. (2003). Somatosensory and motor disturbances in patients with parietal lobe lesions. Advances in Neurology, 93, 179-193.

Gallace, A., \& Spence, C. (2010). The science of interpersonal touch: An overview. Neuroscience and Biobehavioral Reviews, 34, 246-259.

Gallese, V. (2003). The manifold nature of interpersonal relations: The quest for a common mechanism. Philosophical Transactions of the Roval Societv of London B, 358, 517-528.

Gallese, V. (in press). Bodily selves in relation: Embodied simulation as second-person perspective on intersubjectivity. Philosophical Transactions of the Royal Society of London B.

Gallese, V., \& Ebisch, S. (2013). Embodied simulation and touch: The sense of touch in social cognition. Phenomenology \& Mind, 4, 269-291.

Gallese, V., Fadiga, L., Fogassi, L., \& Rizzolatti, G. (2002). Action representation and the inferior parietal lobule. In W. Prinz \& B. Hommel (Eds.), Common mechanisms in perception and action: Attention and performance (pp. 334-355). Oxford, UK: Oxford University Press.

Gallese, V., \& Goldman, A. (1998). Mirror neurons and the simulation theory of mind-reading. Trends in Cognitive Sciences, 2, 493-501.

Gallese, V., \& Sinigaglia, C. (2011). What is so special about embodied simulation? Trends in Cognitive Sciences, 15, 512-519.
Gazzola, V., \& Keysers, C. (2009). The observation and execution of actions share motor and somatosensory voxels in all tested subjects: Single-subject analyses of unsmoothed fMRI data. Cerebral Cortex, 19, 1239-1255.

Gazzola, V., Spezio, M. L., Etzel, J. A., Castelli, F., Adolphs, R., \& Keysers, C. (2012). Primary somatosensory cortex discriminates affective significance in social touch. Proceedings of the National Academv of Sciences. U.S.A., 109, E1657-E1666.

Gibson, J. J. (1962). Observations on active touch. Psvchological Review, 69, 477-491.

Gilbert, S. J., Spengler, S., Simons, J. S., Steele, J. D., Lawrie, S. M., Frith, C. D., et al. (2006). Functional specialization within rostral prefrontal cortex (area 10): A meta-analysis. Iournal of Cognitive Neuroscience, 18, 932-948.

Gilbert, S. J., Williamson, I. D., Dumontheil, I., Simons, J. S., Frith, C. D., \& Burgess, P. W. (2007). Distinct regions of medial rostral prefrontal cortex supporting social and nonsocial functions. Social Cognitive and Affective Neuroscience, 2, 217-226.

Gordon, I., Voos, A. C., Bennett, R. H., Bolling, D. Z., Pelphrey, K. A., \& Kaiser, M. D. (2013). Brain mechanisms for processing affective touch. Human Brain Mabping, 34, 914-922.

Hagen, M. C., Franzén, O., McGlone, F., Essick, G., Dancer, C., \& Pardo, J. V. (2002). Tactile motion activates the human middle temporal/V5 (MT/V5) complex. European Iournal of Neuroscience, 16, 957-964.

Haggard, P. (2008). Human volition: Towards a neuroscience of will. Nature Reviews Neuroscience, 9, 934-946.

Haggard, P., \& Clark, S. (2003). Intentional action: Conscious experience and neural prediction. Consciousness and Cognition, 12, 695-707.

Hertenstein, M. J., Keltner, D., App, B., Bulleit, B. A., \& Jaskolka, A. R. (2006). Touch communicates distinct emotions. Emotion, 6, 528-533.

Hikosaka, O., Tanaka, M., Sakamoto, M., \& Iwamura, Y. (1985). Deficits in manipulative behaviors induced by local injections of muscimol in the first somatosensory cortex of the conscious monkey. Brain Research, 325, 375-380.

Hlushchuk, Y., \& Hari, R. (2006). Transient suppression of ipsilateral primary somatosensory cortex during tactile finger stimulation. Journal of Neuroscience, 26, 5819-5824.

Hughes, G., Desantis, A., \& Waszak, F. (2013). Mechanisms of intentional binding and sensory attenuation: The role of temporal prediction, temporal control, identity prediction, and motor prediction. Psychological Bulletin, 139, 133-151.

Hyvärinen, J. (1982). Posterior parietal lobe of the primate brain. Physiological Reviews, 62, 1060-1129.

Ishida, H., Nakajima, K., Inase, M., \& Murata, A. (2010). Shared mapping of own and others' bodies in visuotactile bimodal area of monkey parietal cortex. Journal of Cognitive Neuroscience, 22, 83-96.

Iwamura, Y., \& Tanaka, M. (1996). Representation of reaching and grasping in the monkey postcentral gyrus. Neuroscience Letters, 214, 147-150.

Jackson, S. R., Parkinson, A., Pears, S. L., \& Nam, S. H (2011). Effects of motor intention on the perception of somatosensory events: A behavioural and functional magnetic resonance imaging study. Quarterlv Iournal of Experimental Psychologv (Hove), 64, 839-854.

Johansson, R. S., \& Flanagan, J. R. (2009). Coding and use of tactile signals from the fingertips in object manipulation tasks. Nature Reviews Neuroscience, 10, 345-359.

Kaneko, T., Caria, M. A., \& Asanuma, H. (1994a). Information processing within the motor cortex. I. Responses of morphologically identified motor cortical cells to stimulation of the somatosensory cortex. Journal of Comparative Neurology, 345, 161-171. 
Kaneko, T., Caria, M. A., \& Asanuma, H. (1994b). Information processing within the motor cortex. II. Intracortical connections between neurons receiving somatosensory cortical input and motor output neurons of the cortex. Iournal of Comparative Neurology, 345, 172-184.

Keysers, C., Kaas, J. H., \& Gazzola, V. (2010). Somatosensation in social perception. Nature Reviews Neuroscience, 11, 417-428.

Knoblich, G., \& Flach, R. (2001). Predicting the effects of actions: Interactions of perception and action. Psvchological Science, 12, 467-472.

Lederman, S. J., \& Klatzky, R. L. (2009). Haptic perception: A tutorial. Attention. Perception, \& Psychophysics, 71, 1439-1459.

Lewis, J. W., \& Van Essen, D. C. (2000). Corticocortical connections of visual, sensorimotor, and multimodal processing areas in the parietal lobe of the macaque monkey. Journal of Comparative Neurology, 428, $112-137$.

Löken, L. S., Wessberg, J., Morrison, I., McGlone, F., \& Olausson, H. (2009). Coding of pleasant touch by unmyelinated afferents in humans. Nature Neuroscience, 12, 547-548.

Morrison, I., Loken, L. S., \& Olausson, H. (2010). The skin as a social organ. Experimental Brain Research, 204, 305-314.

Morrison, I., Tipper, S. P., Fenton-Adams, W. L., \& Bach, P. (2013). "Feeling" others' painful actions: The sensorimotor integration of pain and action information. Human Brain Mapping, 34, 1982-1998.

Nichols, T., Brett, M., Andersson, J., Wager, T., \& Poline, J. B. (2005). Valid conjunction inference with the minimum statistic. Neuroimage, 25, 653-660.

Petrides, M., \& Pandya, D. N. (1984). Projections to the frontal cortex from the posterior parietal region in the rhesus monkey. Journal of Comparative Neurology, 228, 105-116.

Pons, T. P., \& Kaas, J. H. (1986). Corticocortical connections of area 2 of somatosensory cortex in macaque monkeys: A correlative anatomical and electrophysiological study. Iournal of Comparative Neurology, 248, 313-335.

Prinz, W. (2012). Perception and action planning. European Iournal of Cognitive Psychologv, 9, 129-154.

Ramsey, R., Cross, E. S., \& Hamilton, A. F. (2012). Predicting others' actions via grasp and gaze: Evidence for distinct brain networks. Psychological Research, 76, 494-502.

Rizzolatti, G., Fogassi, L., \& Gallese, V. (2002). Motor and cognitive functions of the ventral premotor cortex. Current Opinion in Neurobiology, 12, 149-154.

Rizzolatti, G., \& Sinigaglia, C. (2010). The functional role of the parieto-frontal mirror circuit: Interpretations and misinterpretations. Nature Reviews Neuroscience, 11 264-274.

Roth, M. J., Synofzik, M., \& Lindner, A. (2013). The cerebellum optimizes perceptual predictions about external sensory events. Current Biology, 23, 930-935.

Rozzi, S., Calzavara, R., Belmalih, A., Borra, E., Gregoriou, G. G., Matelli, M., et al. (2006). Cortical connections of the inferior parietal cortical convexity of the macaque monkey. Cerebral Cortex, 16, 1389-1417.
Rozzi, S., Ferrari, P. F., Bonini, L., Rizzolatti, G., \& Fogassi, L. (2008). Functional organization of inferior parietal lobule convexity in the macaque monkey: Electrophysiological characterization of motor, sensory and mirror responses and their correlation with cytoarchitectonic areas. European Iournal of Neuroscience, 28, 1569-1588.

Ruben, J., Schwiemann, J., Deuchert, M., Meyer, R., Krause, T., Curio, G., et al. (2001). Somatotopic organization of human secondary somatosensory cortex. Cerebral Cortex, 11, $463-473$.

Schäfer, K., Blankenburg, F., Kupers, R., Grüner, J. M., Law, I., Lauritzen, M., et al. (2012). Negative BOLD signal changes in ipsilateral primary somatosensory cortex are associated with perfusion decreases and behavioral evidence for functional inhibition. Neuroimage, 59, 3119-3127.

Schippers, M. B., Roebroeck, A., Renken, R., Nanetti, L., \& Keysers, C. (2010). Mapping the information flow from one brain to another during gestural communication. Proceedings of the National Academv of Sciences. U.S.A. 107, 9388-9393.

Schlerf, J., Ivry, R. B., \& Diedrichsen, J. (2012). Encoding of sensory prediction errors in the human cerebellum. Iournal of Neuroscience, 32, 4913-4922.

Sebanz, N., Bekkering, H., \& Knoblich, G. (2006). Joint action: Bodies and minds moving together. Trends in Cognitive Sciences, 10, 70-76.

Shadmehr, R., Smith, M. A., \& Krakauer, J. W. (2010). Error correction, sensory prediction, and adaptation in motor control. Anmual Review of Neuroscience, 33, 89-108.

Shmuel, A., Augath, M., Oeltermann, A., \& Logothetis, N. K. (2006). Negative functional MRI response correlates with decreases in neuronal activity in monkey visual area V1. Nature Neuroscience, 9, 569-577.

Talairach, J., \& Tournoux, P. (1988). Co-planar stereotaxic atlas of the human brain: 3-Dimensional proportional system-An approach to cerebral imaging. New York: Thieme.

van Ede, F., de Lange, F. P., \& Maris, E. (2013). Anticipation increases tactile stimulus processing in the ipsilateral primary somatosensory cortex. Cerebral Cortex. doi:10.1093/cercor/bht111.

Voss, M., Ingram, J. N., Wolpert, D. M., \& Haggard, P. (2008). Mere expectation to move causes attenuation of sensory signals. PLoS One, 3, e2866.

Wang, J., Fan, L., Zhang, Y., Liu, Y., Jiang, D., Zhang, Y., et al. (2012). Tractography-based parcellation of the human left inferior parietal lobule. Neuroimage, 63, 641-652.

Wilson, M., \& Knoblich, G. (2005). The case for motor involvement in perceiving conspecifics. Psychology Bulletin, 131, 460-473.

Wolpert, D. M., \& Flanagan, J. R. (2001). Motor prediction. Current Biology, 11, R729-R732.

Wolpert, D. M., Ghahramani, Z., \& Jordan, M. I. (1995). An internal model for sensorimotor integration. Science 269, 1880-1882.

Wolpert, D. M., Miall, R. C., \& Kawato, M. (1998). Internal models in the cerebellum. Trends in Cognitive Sciences 2, 338-347. 
This article has been cited by:

1. Hiroaki Ishida, Keisuke Suzuki, Laura Clara Grandi. 2015. Predictive coding accounts of shared representations in parieto-insular networks. Neuropsychologia 70, 442-454. [CrossRef] 